\title{
DEGENERATION AND SPECIALIZATION IN ALGEBRAIC FAMILIES OF VECTOR BUNDLES
}

\author{
BY STEPHEN S. SHATZ 1
}

Communicated January 29, 1976

Introduction. If $X$ is a nonsingular $d$-dimensional projective variety (over an algebraically closed field, $k$ ), then by a vector bundle on $X$ we understand a torsion-free, coherent $\mathrm{O}_{X}$-module. The classical notion of vector bundle (= locally free $\mathrm{O}_{X}$-module) is subsumed in the new definition and agrees with it when $X$ is a curve. The new notion is necessary for the study of moduli problems $[\mathbf{G}],[\mathbf{L}]$ and because the classical definition is too rigid for higher dimensional varieties [L]. However, given a vector bundle on $X$, there exists an open subset $U$ of $X$ containing all points of codimension $\leqslant 1$ such that the vector bundle is locally free over $U$. Chern classes can always be defined for such bundles.

We fix, once and for all, a very ample divisor class, $H$, on $X$. Then for any prescheme $S$ over $k$, the line bundle $p^{*} H$ on $X \times_{k} S$ (where $p: X \times_{k} S \rightarrow X$ is the projection) is very ample with respect to $S$. As usual, set $F(n)=F \otimes H^{\otimes n}$, then the Hilbert polynomial $p_{F}(n)$ is defined and, by the Riemann-Roch Theorem, $p_{F}(n)$ has an expression in terms of Chern classes. Indeed, if we set

$$
\operatorname{deg} F=\left(c_{1}(F) \cdot H^{d-1}\right) \quad \text { (intersection no.), }
$$

and refer to $\operatorname{deg} F$ as the degree of $F$ (more properly, the $H$-degree of $F$ ), then

$$
\begin{aligned}
\frac{p_{F}(n)}{\operatorname{rk} F}= & \delta(X) \frac{n^{d}}{d !}+\left(\frac{\operatorname{deg} F}{\operatorname{rk} F}-\frac{\operatorname{deg} K}{2}\right) \frac{n^{d-1}}{(d-1) !} \\
& + \text { terms of lower degree in } n .
\end{aligned}
$$

The quantities $\tilde{\mu}(F)=p_{F}(n) / \mathrm{rk} F, \mu(F)=(\operatorname{deg} F) / \mathrm{rk} F$ are fundamental for what we shall do. The former was introduced by Gieseker, the latter by Takemoto [T], and both were inspired by results of Mumford for curves. We shall concentrate on $\mu$; however, everything we do carries over to $\tilde{\mu}$ suitably interpreted. Call $\mu(F)$ the slope of $F$.

The bundle $F$ is semistable (resp. stable) if for every proper subbundle, $G$, $\mu(G) \leqslant \mu(F)$ (resp. $\mu(G)<\mu(F)$ ). Our bundle $F$ is unstable if it is not semistable. We regard an unstable bundle as "more degenerate" than a semistable bundle, etc.

AMS (MOS) subject classifications (1970). Primary 14F05, 14D20, 14C05.

1 Supported in part, by NSF. 
Main results. Let $F$ be a bundle on $X$, as above. A Harder-Narasimhan Flag for $F$ (abbreviated $\mathrm{HNF}(F)$ ) is a descending chain of subbundles of $F$,

$$
F>F_{t-1}>\cdots>F_{1}>(0)
$$

having the properties:

(a) each factor bundle $F_{j} / F_{j-1}$ is semistable;

(b) $\mu\left(F_{j+1} / F_{j}\right)<\mu\left(F_{j} / F_{j-1}\right), 1 \leqslant j \leqslant t-1$.

(These chains were introduced in $[\mathrm{HN}]$ in the case $X$ was a curve, for other purposes.)

THEOREM 1. Every bundle, $F$, on the d-dimensional, nonsingular, irreducible, projective variety, $X$, possesses a unique HNF. Any two flags (*) satisfying (a) and (b) are identical. Moreover, if $F$ is itself semistable, then $F$ possesses a flag (*) in which

(a) each factor bundle is stable, and

(b) $\mu\left(F_{j+1} / F_{j}\right)=\mu\left(F_{j} / F_{j-1}\right)$.

Theorem 1 is not difficult to prove if one makes full use of the new definition of vector bundle-it is false otherwise. Given a bundle $F$, if we plot in the (rk, deg)-plane the points whose coordinates are the ranks and degrees of the bundles occurring in $(*)$ for $F$, we obtain a polygon which we call the Harder-Narasimhan Polygon for $F(\operatorname{HNP}(F))$. The slopes of the sides of this polygon are exactly the numbers $\mu\left(F_{1}\right), \mu\left(F_{2} / F_{1}\right)$, etc. ocurring in (b) above. Theorem 1 states that every $F$ possesses a unique $\mathrm{HNP}$, and that $\operatorname{HNP}(F)$ is $a$ convex polygon.

Let $X$ be a nonsingular, irreducible, projective $k$-variety and let $S$ be a scheme over $k$. A vector bundle on $X \times_{k} S$, flat over $S$, will be called an algebraic family of vector bundles on $X$ parametrized by $S$. If $F$ is a family on $X$ and $s \in S$, we let $F_{s}$ denote the pull-back of $F$ to the fibre, $X_{s}$, of $X \times_{k} S$ over s. The divisor class $H$ on $X$ induces divisor classes $p^{*} H$ and $H_{s}$ on $X \times{ }_{k} S$ and $X_{s}$, respectively, by pull-back. These are very ample as $H$ is, and semistability is measured via these given very ample sheaves.

THEOREM 2.2 Given $X, S$, and $F$ on $X \times_{k} S$, as above, form $\operatorname{HNP}\left(F_{s}\right)$ for each $s \in S$. If $t_{0} \in S$ is a specialization of $s \in S$, then $H N P\left(F_{t_{0}}\right)$ lies on or above $H N P\left(F_{s}\right)$. That is, the Harder-Narasimhan polygon rises under specialization.

When the moduli scheme for stable bundles exists (i.e., for $X$ a curve [M] or a surface [G]), one can show that $\operatorname{HNP}\left(F_{s}\right)$ is a constructible function of $s$, therefore Theorem 2 implies it is upper semicontinuous. Using the upper semi-

2 I want to thank D. G. Quillen for suggesting that my earlier work for curves, $X$, be formulated as in Theorem 2 -thereby inducing me to prove Theorem 2 in general. 
continuity, we have obtained a fundamental map from vector bundles on $X \times{ }_{k} S$ to collections of $r-1$ algebraic cycles (with nonnegative coefficients) of $S, r$ being the rank of the bundles.

In the simplest case, let $Y$ be a ruled surface with base curve $C$, and let $Y_{c}$ denote the generic fibre. Fix a convex polygon, $P$, with vertices at $(0,0)$, $\left(1, d_{1}\right), \ldots,\left(r, d_{r}\right)$ (the $d$ 's are given integers), and let $\operatorname{Vect}(Y ; P)$ denote the set map of rank $r$ bundles on $Y$ whose HNP at the generic fibre $Y_{c}$ is the given polygon $P$. Then we obtain a

$$
\operatorname{Vect}(Y ; P) \stackrel{\theta}{\longrightarrow} \prod_{r-1 \text { factors }} \operatorname{Hilb}(C) .
$$

It turns out that two bundles on $Y$ have the same image under $\theta$ if and only if their pull-backs to each fibre of $Y$ over $C$ are isomorphic, moreover the map $\theta$ is surjective. These matters are discussed in $\left[\mathbf{S}_{1}\right],\left[\mathbf{S}_{2}\right],\left[\mathbf{S}_{3}\right]$.

\section{REFERENCES}

[G] D. Gieseker, On the moduli of vector bundles on an algebraic surface (preprint).

[HN] G. Harder and R. Narasimhan, The étale cohomology of the moduli of vector bundles over a curve, Invent. Math., 1975.

[L] S. Langton, Valuative criteria for vector bundles, Ann. of Math. (2) 101 (1975), 88-111.

[M] D. Mumford, Projective invariants and projective structures and applications, Proc. Internat. Congr. Mathematicians (Stockholm, 1962), Inst. Mittag-Leffler, Djursholm, 1963, pp. 526-530. MR 31 \#175.

$\left[\mathrm{S}_{1}\right] \mathrm{S}$. Shatz, On the decomposition and specialization of algebraic families of vector bundles (preprint).

$\left[\mathrm{S}_{2}\right] \longrightarrow$ Vector bundles on ruled surfaces (preprint).

$\left[\mathrm{S}_{3}\right] \longrightarrow$ Vector bundles and Hilbert schemes (preprint).

[T] F. Takemoto, Stable vector bundles on algebraic surfaces, Nagoya Math. J. 47 (1972), 29-48. MR 49 \#2735.

DEPARTMENT OF MATHEMATICS, UNIVERSITY OF PENNSYLVANIA, PHILADELPHIA, PENNSYLVANIA 19174 